\title{
Common Fixed Point Theorem in Fuzzy Metric Spaces Under E. A. Like Property
}

\author{
Madhu Shrivastava ${ }^{1}$, K. Qureshi ${ }^{2}$, A. D. Singh $^{3}$ \\ ${ }^{1}$ TIT Group of Instititution, Bhopal, India \\ ${ }^{2}$ Retd. Additional Director, Bhopal, India \\ ${ }^{3}$ Govt. M. V. M. College, Bhopal, India \\ Email address: \\ smadhush1982@gmail.com (M. Shrivastava)
}

\section{To cite this article:}

Madhu Shrivastava, K. Qureshi, A. D. Singh. Common Fixed Point Theorem in Fuzzy Metric Spaces Under E. A. Like Property. Pure and Applied Mathematics Journal. Vol. 5, No. 4, 2016, pp. 141-144. doi: 10.11648/j.pamj.20160504.18

Received: July 4, 2016; Accepted: July 25, 2016; Published: August 21, 2016

Abstract: George and Veeramani [1] modify the concept of fuzzy metric spaces introduced by Kramosil and Michalek [4], Aamri and Moutawakil [8] generalized the notion of non-compatible mapping in metric space by E.A. property.Continuing the above conceptwe prove some commonfixed point theorem for a pair of weakly compatible maps under E.A. Like property.

Keywords: Fuzzy Metric Space, E. A. Property, E. A. Like Property, Weakly Compatible Maps

\section{Introduction}

Fuzzy set theory has various applications in different area. When the notion of fuzzy set was introduced, then it was the turning point in the development of mathematics. It was introduced by Zadeh [7]. Fuzzy set theory has various application in applied science such as neural network theory, stability theory, mathematical programming, modelling theory, engineering science, medical science etc.George and Veeramani [1] modify the concept of fuzzy metric spaces introduced by Kramosil and Michalek [4],with a view to obtain a Hausdorff topology on fuzzy metric spaces, continuously, many authors gives very important results a Sessa [14], Vasuki [12] etc. Aamri and Moutawakil [8] generalized the notion of non-compatible mapping in metric space by E.A. property.It was pointed out in [9] that property E.A. buys containment of ranges without any continuity requirement besides minimizes the commutatively at their point of coincidence.

In this paper, we establish some new results in common fixed point theorems in fuzzy metric spaces under E. A. Like [6].

\section{Definition}

Definition 2.1 [2] A binary operation $*$ : $[0,1] \times[0,1] \rightarrow$ $[0,1]$ is a continuous t-norms if $*$ satisfying conditions:
(1) $*$ is commutative and associative;

(2) $*$ is continuous; if and only if

(3) $a * 1=a$ for all $a \in[0,1]$

(4) $a * b \leq c * d$ whenever $a \leq c$ and $b \leq d$, and $a, b, c, d \in[0,1]$.

Example $2.2 a * b=\min \{a, b\}, a * b=a . b$.

Definition 2.3 [1] A 3-tuple (X, M,*) is said to be a fuzzy metric space if $X$ is an arbitrary set,* is a continuous t-norm and $M$ is a fuzzy set on $X^{2} \times(0, \infty)$ satisfying the following conditions, $\forall x, y, z \in X, s, t>0$ :

(f1) $M(x, y, t)>0$;

$(f 2) M(x, y, t)=1$ if and only if $x=y$.

(f3) $M(x, y, t)=M(y, x, t)$;

(f4) $M(x, y, t) * M(y, z, s) \leq M(x, z, t+s)$

$(f 5) M(x, y,):.(0, \infty) \rightarrow(0,1]$ is continuous.

Then $\mathrm{M}$ is called a fuzzy metric on $\mathrm{X}$. Then $M(x, y, t)$ denotes the degree of nearness between $\mathrm{x}$ and $\mathrm{y}$ with respect to $\mathrm{t}$.

Example 2.4 [1] (Induced fuzzy metric) Let $(X, d)$ be a metric space. Denote $a * b=a b$ for all $a, b \in[0,1]$ and let $M_{d}$ be fuzzy sets on $X^{2} \times(0, \infty)$ defined as follows: $M_{d}(x, y, t)=\frac{t}{t+d(x, y)}$, Then $\left(X, M_{d}, *\right)$ is a fuzzy metric space. We call this fuzzy metric induced by a metric $d$ as the standard intuitionistic fuzzy metric.

Definition 2.5 Two self-mappings $f$ and $g$ of a fuzzy metric space $(X, M, *)$ are called compatible if 
$\lim _{n \rightarrow \infty} M\left(f g x_{n}, g f x_{n}, t\right)=1$ whenever $\left\{x_{n}\right\}$ is a sequence in $\mathrm{X}$ such that

$\lim _{n \rightarrow \infty} f x_{n}=\lim _{n \rightarrow \infty} g x_{n}=x$ for some $x \in X$.

Lemma 2.6 Let $(X, M, *)$ be fuzzy metric space. If there exists $q \in(0,1)$ such that

$M(x, y, q t) \geq M(x, y, t)$ for all $x, y \in X$ and $t>0$, then $x=y$.

Definition 2.7 Let $X$ be a set, $f$ and $g$ self maps of $X$. A point $x \in X$ is called a coincidence point of $f$ and $g$ iff $f x=g x$. We shall call $w=f x=g x$ a point of coincidence of $f$ and $g$.

Definition 2.8 [3] A pair of maps $S$ and $T$ is called weakly compatible pair if they commute at coincidence points.

Definition 2.9 Let $f$ and $g$ be two self-maps of a fuzzy metric $\operatorname{space}(X, M, *)$.we say that $f$ and $g$ satisfy the property E. A. if there exists a sequence $\left\{x_{n}\right\}$ such that, $\lim _{n \rightarrow \infty} f x_{n}=$ $\lim _{n \rightarrow \infty} g x_{n}=z$ for some $z \in X$.

Definition 2.10 Let $f$ and $g$ be two self-maps of a fuzzy metric space $(X, M, *)$. We say that $f$ and $g$ satisfy the property E. A. Like property if there exists a sequence $\left\{x_{n}\right\}$ such that $\lim _{n \rightarrow \infty} f x_{n}=\lim _{n \rightarrow \infty} g x_{n}=z$ for some $z \in$ $f(X)$ or $z \in g(X)$, i. e, $z \in f(X) \cup g(X)$.

Example 2.11 Let $X=[0,2)$ and $M(x, y, t)=\frac{t}{t+d(x, y)}$, for all $x, y \in X$ then $(X, M, *)$ is a fuzzy metric space.

Where $a * b=\min \{a, b\}$.

$$
\begin{aligned}
& A(x)=\left\{\begin{array}{c}
250 \leq x \leq .52 \\
\frac{x}{2} x>.52
\end{array}, S(x)=\left\{\begin{array}{l}
.250 \leq x \leq .6 \\
x-.25 x>.6
\end{array}\right.\right. \\
& T(x)=\left\{\begin{array}{c}
.250 \leq x \leq .6 \\
\frac{x}{4} x>.6
\end{array}, B(x)=\left\{\begin{array}{l}
250 \leq x \leq .95 \\
x-.75 x>.95
\end{array}\right.\right.
\end{aligned}
$$

We define $x_{n}=0.5+\frac{1}{n}$ and $y_{n}=1+\frac{1}{n}$

We have $A(X)=\{0.25\} \cup(.26,1]$,

$$
S(X)=\{0.25\} \cup(0.35,1.75], T(X)=(0.15,0.5]
$$

And $B(X)=\{0.25\} \cup(0.20,1.25]$. $S(X)$

Also limn $\rightarrow \infty A x_{n}=\operatorname{limn} \rightarrow \infty \frac{1}{2}\left[0.5+\frac{1}{n}\right]=0.25 \in$ $\lim n \rightarrow \infty S x_{n}=\operatorname{limn} \rightarrow \infty 0.5+\frac{1}{n}-0.25=0.25 \in A(X)$

$$
\operatorname{limn} \rightarrow \infty T y_{n}=\lim n \rightarrow \infty \frac{1}{4}\left[1+\frac{1}{n}\right]=.25 \in B(X)
$$

And limn $\rightarrow \infty B y_{n}=\lim n \rightarrow \infty 1+\frac{1}{n}-0.75=$ $0.25 \in T(X)$

Definition 2.12 (Common E. A. Property) Let $A, B, S, T: X \rightarrow X$ where $X$ is a fuzzy metric space,then the pair $\{A, S\}$ and $\{B T\}$ said to satisfy common E. A. property if there exist two sequences $\left\{x_{n}\right\}$ and $\left\{y_{n}\right\}$ in $X$ such that $\lim _{n \rightarrow \infty} A x_{n}=$ $\lim _{n \rightarrow \infty} S x_{n}=\lim _{n \rightarrow \infty} T y_{n}=\lim _{n \rightarrow \infty} B y_{n}=z$ for some $z \in X$.

Definition 2.13 (Common E. A. like Property) Let $A, B, S$ and $T$ be self maps of a fuzzy metric space $(X, M, *)$, then the pairs $(A, S)$ and $(B, T)$ said to satisfy common E. A. Like property if there exists two sequences $\left\{x_{n}\right\}$ and $\left\{y_{n}\right\}$ in $\mathrm{X}$ such that

$$
\lim _{n \rightarrow \infty} A x_{n}=\lim _{n \rightarrow \infty} S x_{n}=\lim _{n \rightarrow \infty} T y_{n}=
$$

$\lim _{n \rightarrow \infty} B y_{n}=z$

Where $z \in S(X) \cap T(X)$ or $z \in A(X) \cap B(X)$.

Role of E.A. property in proving common fixed point theorems can be concluded by following,

(1) It buys containment of ranges without any continuity requirements.

(2) It minimizes the commutativity conditions of the maps to the commutativity at their points of coincidence.

(3) It allows replacing the completeness requirement of the space with a more natural condition of closeness of the range.

Of course, if two mappings satisfy E. A. like property then they satisfy E. A. property also, but, on the other hand, E. A. like property relaxes the condition of containment of ranges and closeness of the ranges to prove common fixed point theorems, which are necessary with E. A. property.

\section{Main Results}

Theorem-(3.1) - Let $f$ and $g$ be self-maps of a fuzzy metric space $(X, M, *)$, satisfying $M(x, y, t)>0$ for all $x, y$ in $X$ and $t>0$, such that following condition holds-(I) $M(f x, f y, t) \geq r\left(\max \left\{\begin{array}{c}M(f x, g y, t), M(g x, f y, t) \\ , M(g x, g y, t)\end{array}\right\}\right)$,

for all $x, y \in X$

(II) $f$ and $g$ Satisfy the E.A. Like property.

Where $r:[0,1] \rightarrow[0,1]$ is a continuous function such that $r(t)>t$ for each $0<t<1, r(0)=0$ and $r(1)=1$.

Then there exist a unique common fixed point of $f$ and $g$.

Proof - Since $f$ and $g$ satisfy E. A. Like property. Therefore there exists a sequence $\left\{x_{n}\right\}$ in $X$,

Such that

$\lim _{n \rightarrow \infty} f x_{n}=\lim _{n \rightarrow \infty} g x_{n}=z \in f(x)$ or $g(X)$. Suppose that $\lim _{n \rightarrow \infty} f x_{n}=z \in g(X)$,

Therefore $z=g u$ for some $u \in X$. Now we show that $f u=g u$, from $(\mathrm{I})$, we have

$$
M\left(f u, f x_{n}, t\right) \geq r\left(\max \left\{\begin{array}{c}
M\left(f u, g x_{n}, t\right), M\left(g u, f x_{n}, t\right), \\
M\left(g u, g x_{n}, t\right)
\end{array}\right\}\right)
$$

Taking $\lim n \rightarrow \infty$, we get.

$$
\begin{gathered}
M(f u, z, t) \geq r\left(\max \left\{\begin{array}{c}
M(f u, z, t), M(g u, z, t), \\
M(g u, z, t)
\end{array}\right\}\right) \\
=r(\max \{M(f u, z, t), M(z, z, t), M(z, z, t)\}) \\
=r(M(z, z . t))=r(1)=1
\end{gathered}
$$

This implies that $f u=z=g u$. i.e $u$ is coincidence point of $f$ and $g$.

Since $f$ and $g$ are weakly compatible. Therefore $f z=$ $f g u=g f u=g z$

Now we show that $f z=z$, If not from (I), we have $M\left(f z, f x_{n}, t\right) \geq r\left(\max \left\{\begin{array}{c}M\left(f z, g x_{n}, t\right), M\left(g z, f x_{n}, t\right), \\ M\left(g z, g x_{n}, t\right)\end{array}\right\}\right)$

Takin glim $n \rightarrow \infty$,we get.

$$
\begin{aligned}
& M(f z, z, t) \geq r\left(\max \left\{\begin{array}{c}
M(f z, z, t), M(g z, z, t), \\
M(g z, z, t)
\end{array}\right)\right. \\
& M(f z, z, t) \geq r\left(\max \left\{\begin{array}{c}
M(f z, z, t), M(f z, z, t), \\
M(f z, z, t)
\end{array}\right\}\right)
\end{aligned}
$$




$$
M(f z, z, t) \geq r(M(f z, z, t))>M(f z, z, t)
$$

Which is a contradiction. Hence $f z=g z=z$. Hence $z$ is a common fixed point of $f$ and $g$.

Uniqueness - Let $z_{1}$ be another fixed point of and $g$, such that $z_{1} \neq z$, then from (I),we have

$$
\begin{gathered}
M\left(f z, f z_{1}, t\right) \geq r\left(\max \left\{\begin{array}{c}
M\left(f z, g z_{1}, t\right), M\left(g z, f z_{1}, t\right), \\
M\left(g z, g z_{1}, t\right)
\end{array}\right)\right) \\
M\left(z, z_{1}, t\right) \geq r\left(\max \left\{M\left(z, z_{1}, t\right), M\left(z, z_{1}, t\right), M\left(z, z_{1}, t\right)\right\}\right) \\
M\left(z, z_{1}, t\right) \geq r\left(M\left(z, z_{1}, t\right)\right)>M\left(z, z_{1}, t\right)
\end{gathered}
$$

Which is a contradiction. Hence $z=z_{1}$

Theorem-3.2 $A, B, S, T$ be self-maps of a fuzzy metric $\operatorname{space}(X, M, t)$ satisfying the following condition-

(I) $M(A x, B y, t) \geq r(\max \{M(S x, T y, t), M(B y, S x, t)$,

$$
\begin{gathered}
M(A x, T y, t), M(S x, A x, t), \\
\frac{a \cdot M(A x, B y, t)+b \cdot M(A x, T y, t)}{a \cdot M(S x, B y, t)+b \cdot M(S x, T y, t)} \\
\left.\left.\frac{\text { c. } M(S x, B y, t)+d \cdot M(S x, T y, t)}{c \cdot M(B y, T y, t)+d}\right\}\right)
\end{gathered}
$$

(II)-Pairs $(A, S)$ and $(B, T)$ are weakly compatible.

(III)-Pairs $(A, S)$ and $(B, T)$ satisfying common E. A. Like property.

for all $x, y$ in $X$ and $t>0$, where $a$ and $b(\mathrm{c}$ and d) can not be simultaneous 0 , and $a, b, c, d \geq 0$

Then A,B,S,T have a unique common fixed point.

Proof - Since $(A, S)$ and $(B, T)$ satisfy common E. A. Like property, therefore there exist two sequences $\left\{x_{n}\right\}$ and $\left\{y_{n}\right\}$ in $X$, such that

$$
\lim _{n \rightarrow \infty} A x_{n}=\lim _{n \rightarrow \infty} S x_{n}=\lim _{n \rightarrow \infty} T y_{n}=\lim _{n \rightarrow \infty} B y_{n}=z
$$

Where $z \in S(X) \cap T(X)$ or $z \in A(X) \cap B(X)$

Suppose that $z \in S(X) \cap T(X)$, Now We have $\lim _{n \rightarrow \infty} A x_{n}=z \in S(X)$, then $z=S u$ for some $u \in X$ Now we claim that $A u=S u$,from (I), We have

$$
\begin{gathered}
M\left(A u, B y_{n}, t\right) \geq r\left(\operatorname { m a x } \left\{M\left(S u, T y_{n}, t\right), M\left(B y_{n}, S u, t\right),\right.\right. \\
M\left(A u, T y_{n}, t\right), M(S u, A u, t), \\
\frac{a \cdot M\left(A u, B y_{n}, t\right)+b \cdot M\left(A u, T y_{n}, t\right)}{a \cdot M\left(S u, B y_{n}, t\right)+b \cdot M\left(S u, T y_{n}, t\right)} \\
\left.\left.\frac{c \cdot M\left(S u, B y_{n}, t\right)+d \cdot M\left(S u, T y_{n}, t\right)}{c \cdot M\left(B y_{n}, T y_{n}, t\right)+d}\right\}\right)
\end{gathered}
$$

$\lim n \rightarrow \infty$,we get

$$
\begin{gathered}
M(A u, z, t) \geq r(\max \{M(z, z, t), M(z, z, t), M(A u, z, t), \\
M(z, A u, t), \frac{a \cdot M(A u, z, t)+b \cdot M(A u, z, t)}{a \cdot M(z, z, t)+b \cdot M(z, z, t)}, \\
\left.\left.\frac{c \cdot M(z, z, t)+d \cdot M(z, z, t)}{c \cdot M(z, z, t)+d}\right\}\right)
\end{gathered}
$$

$$
\begin{gathered}
M(A u, z, t) \geq r\left(\max \left\{\begin{array}{c}
1,1, M(A u, z, t), M(A u, z, t), \\
M(A u, z, t), 1
\end{array}\right\}\right) \\
M(A u, z, t) \geq r(1)=1
\end{gathered}
$$

Hence $A u=z=S u$, Now We have $\lim _{n \rightarrow \infty} B x_{n}=z \in$ $S(X)$, then $T v=z$, for some $v \in X$

Now we claim that $T v=B v$, from (I), We have

$$
\begin{gathered}
M\left(A x_{n}, B v, t\right) \geq r\left(\operatorname { m a x } \left\{M\left(S x_{n}, T v, t\right), M\left(B v, S x_{n}, t\right),\right.\right. \\
M\left(A x_{n}, T v, t\right), M\left(S x_{n}, A x_{n}, t\right), \\
\frac{a \cdot M\left(A x_{n}, B v, t\right)+b \cdot M\left(A x_{n}, T v, t\right)}{a \cdot M\left(S x_{n}, B v, t\right)+b \cdot M\left(S x_{n}, T v, t\right)} \\
\left.\left.\frac{c \cdot M\left(S x_{n}, B v, t\right)+d \cdot M\left(S x_{n}, T v, t\right)}{c \cdot M(B v, T v, t)+d}\right\}\right)
\end{gathered}
$$

$\lim n \rightarrow \infty$, we get

$$
\begin{gathered}
M(z, B v, t) \geq r(\max \{M(z, z, t), M(B v, z, t), M(z, z, t), \\
M(z, z, t), \frac{a \cdot M(z, B v, t)+b \cdot M(z, z, t)}{a \cdot M(z, B v, t)+b \cdot M(z, z, t)}, \\
\left.\left.\frac{c \cdot M(z, B v, t)+d \cdot M(z, z, t)}{c \cdot M(B v, z, t)+d}\right\}\right) \\
M(z, B v, t) \geq r\left(\max \left\{\begin{array}{c}
1, M(B v, z, t), 1,1, \\
1,1
\end{array}\right\}\right) \\
M(z, B v, t) \geq r(1)=1
\end{gathered}
$$

Hence $B v=z=T v$

Since the pair $(A, S)$ is weakly compatible, therefore $A z=A S u=S A u=S z$.

Now we show that $A z=z$,

$$
M\left(A z, B y_{n}, t\right)
$$$$
\geq r\left(\operatorname { m a x } \left\{M\left(S z, T y_{n}, t\right), M\left(B y_{n}, S z, t\right), M\left(A z, T y_{n}, t\right),\right.\right.
$$

$$
\begin{gathered}
M(S z, A z, t), \frac{a \cdot M\left(A z, B y_{n}, t\right)+b \cdot M\left(A z, T y_{n}, t\right)}{a \cdot M\left(S z, B y_{n}, t\right)+b \cdot M\left(S z, T y_{n}, t\right)}, \\
\left.\left.\frac{\text { c. } M\left(S z, B y_{n}, t\right)+d \cdot M\left(S z, T y_{n}, t\right)}{c \cdot M\left(B y_{n}, T y_{n}, t\right)+d}\right\}\right)
\end{gathered}
$$

$\lim n \rightarrow \infty$,we get

$$
M(A z, z, t) \geq
$$

$r(\max \{M(A z, z, t), M(z, A z, t), M(A z, z, t), M(A z, A z, t)$,

$$
\begin{gathered}
\frac{a \cdot M(A z, z, t)+b \cdot M(A z, z, t)}{a \cdot M(A z, z, t)+b \cdot M(A z, z, t)} \\
\left.\left.\frac{c \cdot M(A z, z, t)+d \cdot M(A z, z, t)}{c \cdot M(z, z, t)+d}\right\}\right) \\
M(A z, z, t) \geq r\left(\max \left\{\begin{array}{c}
M(A z, z, t), M(z, A z, t), M(A z, z, t), \\
1,1, \\
M(A z, z, t)
\end{array}\right\}\right)
\end{gathered}
$$




$$
M(A z, z, t) \geq r(1)=1
$$

Hence $A z=z=S z$

Since the pair $(B, T)$ is weakly compatible, therefore $B z=B T v=T B v=T z$.

Now we show that $B z=z$,

$$
\begin{gathered}
\geq r\left(\operatorname { m a x } \left\{M\left(S x_{n}, T z, t\right), M\left(B z, S x_{n}, t\right), M\left(A x_{n}, T z, t\right),\right.\right. \\
M\left(S x_{n}, A x_{n}, t\right), \frac{a \cdot M\left(A x_{n}, B z, t\right)+b \cdot M\left(A x_{n}, T z, t\right)}{a \cdot M\left(S x_{n}, B z, t\right)+b \cdot M\left(S x_{n}, T z, t\right)}, \\
\left.\left.\frac{c \cdot M\left(S x_{n}, B z, t\right)+d \cdot M\left(S x_{n}, T z, t\right)}{c \cdot M(B z, T z, t)+d}\right\}\right)
\end{gathered}
$$

$\lim n \rightarrow \infty$,we get

$$
\begin{gathered}
M(z, B z, t \geq r(\max \{M(z, B z, t), M(B z, z, t), M(z, B z, t), \\
M(z, z, t), \frac{a \cdot M(z, B z, t)+b \cdot M(z, B z, t)}{a \cdot M(z, B z, t)+b \cdot M(z, B z, t)}, \\
\left.\left.\frac{c \cdot M(z, B z, t)+d \cdot M(z, B z, t)}{c \cdot M(B z, B z, t)+d}\right\}\right) \\
M(z, B z, t) \geq r\left(\max \left\{\begin{array}{c}
M(z, B z, t), M(B z, z, t), \\
M(z, B z, t), 1,1, M(z, B z, t)
\end{array}\right\}\right) \\
M(z, B z, t) \geq r(1)=1
\end{gathered}
$$

Hence $B z=z=T z$.

Thus $z$ is common fixed pointof $A, B, S$ and $T$.

Uniqueness - Suppose that $z_{1}$ is another common fixed point of $A, B, S$ and $T$.

such that $z_{1} \neq z$. then from (I)

$$
\begin{gathered}
M\left(A z, B z_{1}, t\right) \geq r\left(\operatorname { m a x } \left\{M\left(S z, T z_{1}, t\right), M\left(B z_{1}, S z, t\right),\right.\right. \\
M\left(A z, T z_{1}, t\right), M(S z, A z, t), \\
\frac{a \cdot M\left(A z, B z_{1}, t\right)+b \cdot M\left(A z, T z_{1}, t\right)}{a \cdot M\left(S z, B z_{1}, t\right)+b \cdot M\left(S z, T z_{1}, t\right)} \\
\left.\left.\frac{c \cdot M\left(S z, B z_{1}, t\right)+d \cdot M\left(S z, T z_{1}, t\right)}{c \cdot M\left(B z_{1}, T z_{1}, t\right)+d}\right\}\right) \\
M\left(z, z_{1}, t\right) \geq r\left(\operatorname { m a x } \left\{M\left(z, z_{1}, t\right), M\left(z_{1}, z, t\right), M\left(z, z_{1}, t\right),\right.\right. \\
M(z, z, t), \frac{a \cdot M\left(z, z_{1}, t\right)+b \cdot M\left(z, z_{1}, t\right)}{a \cdot M\left(z, z_{1}, t\right)+b \cdot M\left(z, z_{1}, t\right)} \\
\left.\left.\frac{c \cdot M\left(z, z_{1}, t\right)+d \cdot M\left(z, z_{1}, t\right)}{c \cdot M\left(z_{1}, z_{1}, t\right)+d}\right\}\right) \\
M\left(z, z_{1}, t\right) \geq r\left(\operatorname { m a x } \left\{M\left(z, z_{1}, t\right), M\left(z_{1}, z, t\right),\right.\right. \\
\left.\left.M\left(z, z_{1}, t\right), 1,1, M\left(z, z_{1}, t\right)\right\}\right) \\
M\left(z, z_{1}, t\right) \geq r(1)=1
\end{gathered}
$$

Hence $z=z_{1}$

Theorem 3.3 Let $A, B, S$ and $T$ be self maps of a fuzzy metric space $(X, M, *)$ satisfying the following conditions:

$(I) A(X) \subset T(X)$ and $B(X) \subset S(X)$,

$$
\text { (II) } M\left(A x, B y, \geq \max \left(r\left\{\begin{array}{c}
M(S x, T y, t), M(A x, S x, t), \\
M(B y, T y, t), \\
M(S x, B y, t), M(A x, T y, t)
\end{array}\right\}\right)\right.
$$

for all $x, y$ in $X$ and $t>0$,

(III) Pairs $(A, S)$ or $(B, T)$ satisfy E. A. property,

(IV) Pairs $(A, S)$ and $(B, T)$ are weakly compatible.

If the range of one of $A, B, S$ and $T$ is a closed subset of $X$, then $A, B, S$ and $T$ have a common fixed point in $X$

Proof- Proof as above.

\section{References}

[1] A. George and P. Veeramani. On some results in fuzzy metric spaces. Fuzzy Sets and Systems, 64: 395: 399, 1994.

[2] B. Schweizer and ASklar. Probabilistic Metric Spaces. North Holland, 1983.

[3] G. Jungck and B. E. Rhoades. Fixed point for set valued functions without continuity. Indian J. Pure and Appl. Math., 29(3): 227: 238, 1998.

[4] I. Kramosil and J. Michalek. Fuzzy metric and statistical metric spaces. Kybernetika, 15: 326: 334, 1975.

[5] J. X. Fang and Y. Gao. Common_xed point theorems under strict contractive conditions in menger spaces. Nonlin. Anal. (TMA), 70(1): 184: 193, 2009.

[6] Kamal Wadhwa, HariomDubeyand Reena Jain,Impact of E. A. Like" Property on Common Fixed Point Theorems in Fuzzy Metric Spaces, Journal of Advanced Studies in Topology, Vol. 3, No. 1, 2012, 52-59.

[7] L. A. Zadeh. Fuzzy sets. Infor. Control, 18: 338: 353, 1965.

[8] M. Aamri and D. El Moutawakil. Some new common fixed point theorems under strict contractive conditions. J. Math. Anal.Appl., 27: 181: 188, 2002.

[9] M. Imdad and J. Ali. Some common fixed point theorems in fuzzy metric spaces. Math. Commun., 11: 153: 163, 2006.

[10] P. Vijayaruju and Z. M. I. Sajath. Some common fixed point theorems in fuzzy metric spaces. Inter. J. of Math. Anal., 3(15): 701: 710, 2009.

[11] R. P. Pant. Common fixed point for non-commuting mappings. J. Math. Anal. Appl., 188: 436: 440, 1994.

[12] R. Vasuki. Commonfixed points for R-weakly commuting maps in fuzzy metric spaces. Indian J. Pure Appl. Math., 30: 419: 423,1999.

[13] S. Kumar. Insert the title of the paper. J. Appl. Math. Inform., 29(1-2): 395: 405, 2011.

[14] S. Sessa. On a weak commutativity of mappings in fixed point considerations. Publ. Inst. Math., 32(46): 149: 153, 1982.

[15] V. Pant. Some fixed point theorems in fuzzy metric spaces. Tamkang J. of Math., 40: 59: 66, 2009. 